\title{
Personalized Home Healthcare Options for Smart Service Delivery and Patient-Centered Monitoring
}

\author{
Adriana Alexandru and Marilena Ianculescu
}

National Institute for R\&D in Informatics, Bucharest, Romania

Correspondence should be addressed to: Adriana Alexandru; adriana.alexandru@ici.ro

Received date: 6 March 2018; Accepted date: 8 March2018; published date: 12 June 2019

Academic Editor: Patrik Pucer

Copyright (C) 2019. Adriana Alexandru and Marilena lanculescu. Distributed under Creative Commons CC-BY 4.0

\begin{abstract}
Adapting the patients' monitoring in a familiar and age-friendly habitat has lately become a blustering necessity in the context of the global ageing of the population (due to the increasing longevity and the average decreased birth rates), associated with co-morbidities and degenerative dysfunctionalities. As the number of elderly mounts, the number of available or appropriate caretakers diminishes, the healthcare and social systems need increased financial and human resources. In the same time, the people are more and more accustomed with the use of digital technology and eager to use it in order to be better informed and empowered regarding the management of their own health, to have broader access to customized health services and, last but not least, to integrate it in their everyday life for preserving as much as possible a healthy, independent and active life apart from an institutionalized environment. Smart technology and devices are extending the performances of the continuously emerging health informatics that are directly involved in providing personalized monitoring and healthcare services that target a specific patient and are adapted to his home, needs and specificities. This paper aims to demonstrate how an existing health informatics system - the PROlonging ACTIVE life for an independent and healthy AGEING (ProActive Ageing) integrated platform for online services - can be improved and developed into an intelligent decision support system with real-time locating facilities in order to provide a better support to elderly to live at home and to have real-time access to smart monitoring and service delivery.
\end{abstract}

Keywords: IoT, patient monitoring, Intelligent Decision Support Systems, Big Data Analytics.

Cite this Article as: Adriana Alexandru and Marilena Ianculescu (2019)," Personalized Home Healthcare Options for Smart Service Delivery and Patient-Centered Monitoring", Journal of e-health Management, Vol. 2019 (2019), Article ID 630156, DOI: 10.5171/2019.630156 


\section{Introduction}

Taking into consideration the global aging of population, the growing demands for material and financial resources in health

and social systems, as well as the growing accountability of citizens to their own health, it has become necessary to implement a new model of patientcentered health care that it is based on a clear identification of the specific needs, living conditions and life experiences of a given patient, and real communication between healthcare professionals and patient involvement in the management of their own health.

The convergence between innovative digital technologies and healthcare services has the ability to boost and achieve better access to care, increased treatment options, and the creation of new opportunities for personalized and patient-centered medical acts. New smart devices and technologies that allow real-time gathering of medical / environmental data and information, and the availability of massive amounts of patient-related data provide new ways to deliver personalized health care and health care services. One way to support migration to the patient-centered health care model is to monitor and integrate a patient's medical data collected in and out of a clinical environment in treatment protocols, clinical research studies, remote monitoring programs, and preventive actions and measures.

\section{Basic Concepts}

\section{Intelligent Decision Support Systems (IDSS)}

A clinical Decision Support System (DSS) is a software designed to perform data analysis and help health care professionals in making clinical decisions. DSS has the role of increasing the quality of care, enhancing health outcomes and reducing human errors while improving efficiency, costbenefit ratio and patient satisfaction. These systems include basic tools for assessment, and monitoring (for example vital signs monitoring), informative tools for providing computerized alerts, reminders and electronic clinical guidelines, and advanced diagnosis and advisory tools which use statistics, machine learning and artificial intelligent techniques to provide better insights by using the collected data.

When dealing with Intelligent Decision Support Systems (IDSS), the system provides intelligent decision support that helps in decision-making through intelligent behaviour that may include learning and reasoning that can be achieved through implementing rule-based expert systems, knowledge-based systems or neural network systems (Basu et al., 2012)

\section{The Internet of Things}

The Internet of Things (IoT) is the network of physical objects or "things" that incorporate electronic devices, software technologies, sensors and network connectivity that make it easier for these objects to collect and exchange data to benefit from various services. IoT is defined as "a dynamic global network infrastructure with self-configuring capabilities based on standard and interoperable communication protocols where physical and virtual "things" have identities, physical attributes, and virtual personalities and use intelligent interfaces, and are seamlessly integrated into the information network"(IERC 2014). Building the IoT is considered to be the next industrial revolution under the heading of Industry 4.0 (Kagermann et al., 2013; Mantea et al., 2016).

Health and medical care are the most attractive areas of application for this concept. IoT is used in many medical applications, such as remote health monitoring, health programs, chronic ageing-related diseases and elderly care. Different medical devices, sensors, diagnostic and imaging devices can be seen as smart devices or objects that are an essential part of the IoT. Actual wirelessbased healthcare networks support the management of chronic ageing-related 
diseases and elderly, early diagnosis, realtime monitoring, and medical emergencies.

In IoT-based health systems, the main technologies used are:

- Sensors that collect patient data;

- Microcontrollers that process, analyze and provide wireless data communications;

- Microprocessors that enable rich graphical user interfaces;

- Specific health gateways through which sensor data is analyzed and sent to Cloud.

\section{Sensing as a Service Concept (SNaaS)}

- The implementation of IoT technology for data collection is a technically difficult task for interested organizations. The "sensing as a service (SNaaS)" concept, developed by ICI Bucharest, aims to facilitate the access of these organizations to the collected data and to the results of their processing, without involving them in the technical aspects related to the implementation and maintenance of the IoT system (Neagu et al., 2016).

- From a technological point of view, SNaaS conceptually exploits the complementarity of IoT and Cloud computing technologies on: computing and storage capacity (limited vs. virtually unlimited), components involved (real things versus virtual resources), coverage (limited vs. omnipresent), Internet use (data concentration and transmission versus service delivery). This complementarity generates obvious benefits when integrated: scalable capabilities and cloud resources compensate the IoT technological limitations, while IoT ability to interact with real-world objects allows diversification of cloud-based services.

- SNaaS is based on the role of the sensing service provider, which builds its offer to the beneficiary organizations by integrating specialized services, such as providing and maintaining the IoT devices, collecting and publishing in the cloud the data available from the IoT infrastructure, processing and advanced analysis of IoT data, ensuring access to data and information for different categories of users within the beneficiary organization. The role of these service providers is played by specialized firms on a contractual basis, but some of these services can even be implemented by the SNaaS provider.

\section{Big Data and Big Data Analytics in Health}

In the context of the use of IoT in the health sector, huge amounts of data are generated. Medical data sources are continually evolving and combining sets of data leading to even greater complexity and creating new exploitation possibilities. "Big Data in Health refers to large routinely or automatically collected datasets, which are electronically captured and stored. It is reusable in the sense of multipurpose data and comprises the fusion and connection of existing databases for the purpose of improving health and health system performance. It does not refer to data collected for a specific study." (Manyika et al., 2011).

In the context of medical care, Big Data Analytics (BDA) is defined as the ability to acquire, store, process, and analyze a large amount of structured, semi-structural or unstructured health data and provide users with useful and meaningful information that enables them to discover new knowledge in a timely manner (Ianculescu et al., 2017a). BDA applications aim to improve patient safety and clinical outcomes, while increasing disease management (IBM, 2012). They represent a huge opportunity to extract useful information for taking the best measures at the level of the whole population.

Building a dynamic, comprehensive, complex, easily accessible and scalable profile associated with a particular patient has the potential to identify the deficient 
aspects of providing health services tailored to its specificities and provides a more comprehensive perspective of proactive and preventive medical acts.

\section{Case study: Proactive Ageing platform}

The concept of "centricity-per-patient" means designing a medical service with the patient as the core nucleus. This can bring many challenges for senior patients, especially those with multiple chronic ageing-related conditions.

Health informatics applications can provide a viable solution as they have considerable potential for improving the quality of geriatrics and gerontology by providing new facilities and opportunities to support integrated health services, independent elderly life, and medical research.

The PROlonging ACTIVE life for an independent and healthy AGEING (ProActive Ageing) project focuses on developing online tools for (Ianculescu et al., 2017b):

- A sustainable (re)integration of ageing persons in social and working life.

- Enhancing the own health and independence of an ageing person, with a strong emphasis on lifelong learning, knowledge sharing.

- Providing comprehensive training courses addressed formal carers of ageing persons.

- Achieving more efficient methods of delivering improved outcomes from healthcare research, good practice and a better service to patients.

Taking into consideration the global ageing of the population, the ProActive Ageing integrated platform for online services developed inside the project promotes a better social inclusion of ageing people by providing enhanced IT-based services that aim to facilitate and promote their access to long-life education. In order to help ageing people, medical staff and caregivers, the system offers practical, motivating, reliable and friendly informatics supportable to empower seniors by providing more "human-centered" social and health systems tools. Moreover, the project supports healthcare providers to provide instruments for specific strategies, protocols and methods for maintenance of an adequate level of biologic and social functions of ageing people (Ianculescu and Alexandru, 2016).

The potential beneficiaries of the ProActive Ageing integrated platform for online services are:

- The Ageing citizens: by providing comprehensive information on ageing issues, a better management of chronic ageing-related diseases, supporting in managing daily activities through the services provided by the platform and sustaining them to obtain a prolonged independence and an increase in the time they can live at home.

- The Patients with chronic ageingrelated diseases: ProActive Ageing can contribute to obtain a longer period of time in which they live and work independently, and that will lead to avoidance of early institutionalization, resulting in a reduced demand for public health organizations.

- The Health professionals from ageing-related specialties: the efficiency of the healthcare services that target the ageing patients can be improved due to the broader access to health information which is facilitated by the ProActive Ageing platform.

- The Social services focusing both on ageing people and elderly, and formal carers: The health care services provided by the ProActive Ageing platform can also be integrated with social services in order to obtain a better communication and coordination of the activities of carers, social services and family members.

ProActive Ageing is an integrated platform (see Figure 1) for online services. 


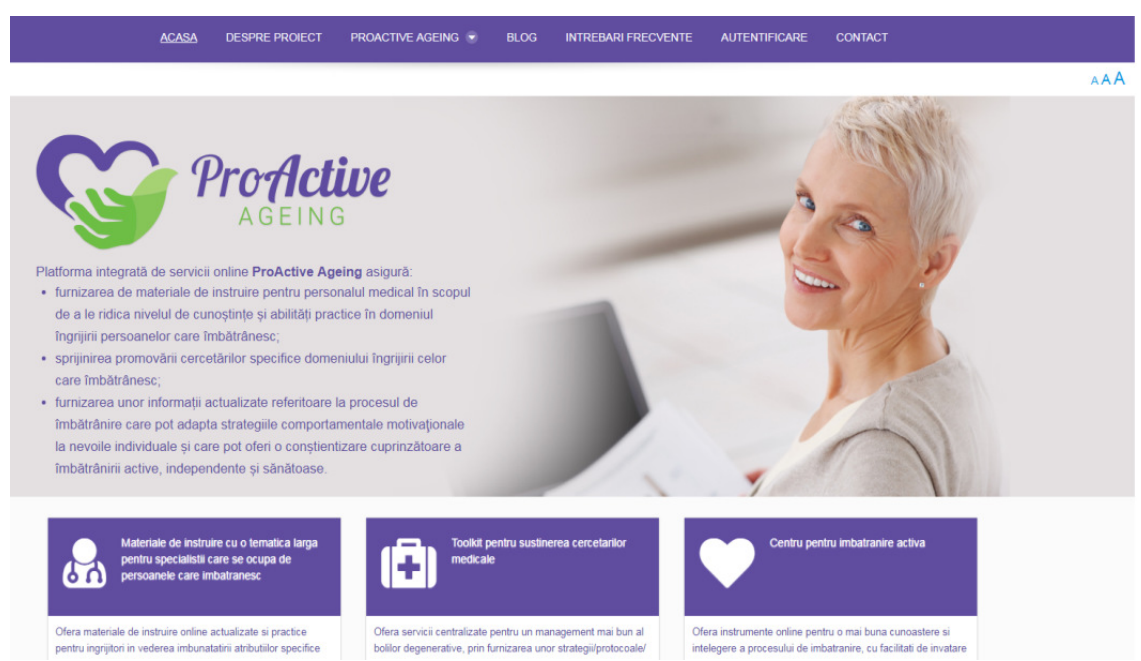

Fig. 1: Home page of Proactive ageing platform

The IT-based services are structured in three thematic modules that provide practical and motivating tools that are able to support the social interaction and adaptability of individuals; social systems and health care to the increasing ageing population (see Table 1).

Table 1: Brief description of the thematic modules of the Proactive ageing integrated platform

\begin{tabular}{|l|l|}
\hline \multicolumn{1}{|c|}{ Name of thematic module } & \multicolumn{1}{c|}{ Description } \\
\hline Centre for Active Ageing & $\begin{array}{l}\text { It aims to provide support for seniors and their } \\
\text { families to have access to appropriate information } \\
\text { regarding a diverse range of age-related issues } \\
\text { like adapting their homes, age-degenerative } \\
\text { diseases, legal aspects, healthy way of living etc. } \\
\text { (see Figure 2) }\end{array}$ \\
\hline $\begin{array}{l}\text { Comprehensive training materials } \\
\text { for specialists taking care of ageing } \\
\text { people }\end{array}$ & $\begin{array}{l}\text { It aims to provide up-to-date curricula that } \\
\text { highlight the specific healthcare addressing the } \\
\text { seniors. }\end{array}$ \\
\hline $\begin{array}{l}\text { A Toolkit to Support Medical } \\
\text { Research Addressed to Healthy and } \\
\text { Active Ageing }\end{array}$ & $\begin{array}{l}\text { Its scope is to sustain the ageing-related health } \\
\text { specialists by providing a practical framework } \\
\text { able to support the communication and research. }\end{array}$ \\
\hline
\end{tabular}




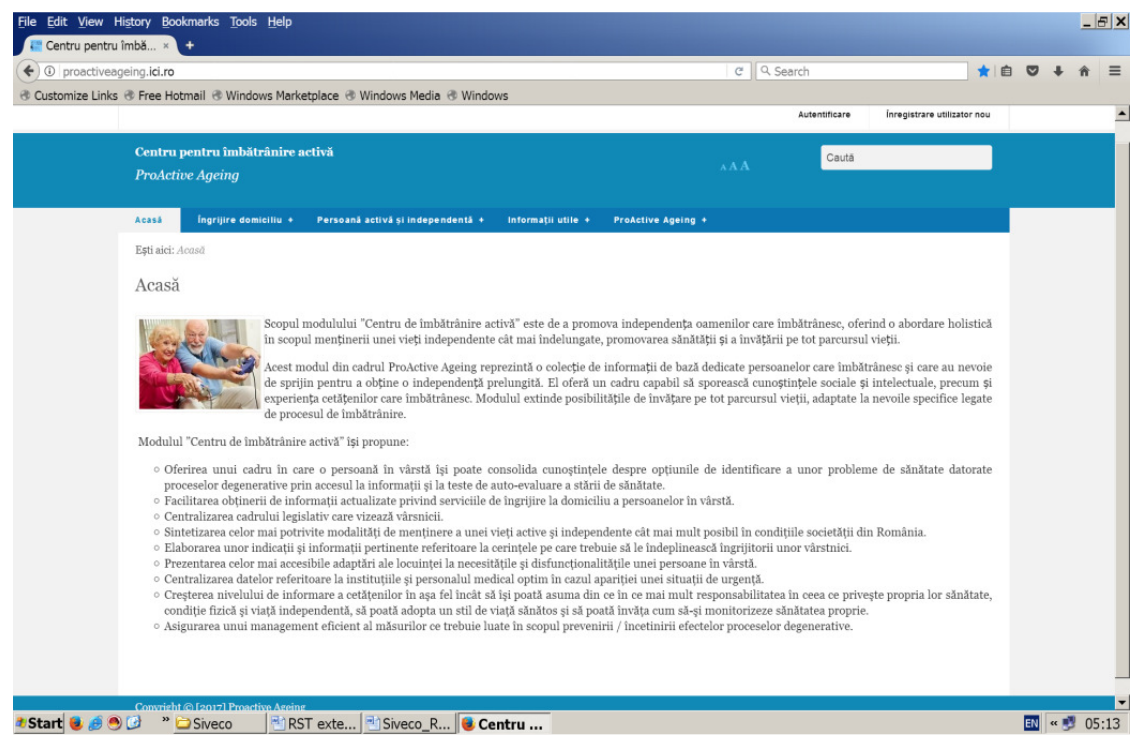

Fig. 2: Home page of "Center for Active Ageing"

The functional model of the ProActive Ageing integrated platform for online services is embedded in those IT tools that support a citizen / patient-centered approach to both management of the state of health and an independent and active life of the elderly, as well as medical research in geriatrics, gerontology or other medical specialities related to the ageing process. At the same time, the platform puts at the disposal of instructors and caregivers of older people a tool that provides a comprehensive framework for updating (according to current trends) knowledge regarding personalized ways of taking care of seniors.

The online person-centric tools for a better knowledge and understanding of the ageing process, with facilities for lifelong learning, designed for people who are ageing and who want to stay active, healthy and independent can have a real impact on:

- the personal autonomy, quality of life and health,

- delaying the institutionalization of ageing people,

- the improvement and better understanding of the duties assigned to medical professionals who deal directly with the care of the ageing,
- increasing the quality of research and health care inside the anti-ageing domain by customizing and integrating the management of chronic ageingrelated diseases with other health services and for achieving an independent living.

ProActive Ageing integrated platform for online services can be further upgraded with new components able to provide new functionalities that aim to support a realtime monitoring of the senior patient in his/her familiar environment or in an institutionalized one. The new functionalities are designed to add preventive and pro-active characteristics to the services provided by the platform and to transform it into a practical and motivating smart technology application.

ProActive Ageing platform is currently updated into an Intelligent Decision Support Systems (IDSS) by integrating new components able to provide patient-centric oriented services.

\section{Description of the proposed intelligent decision support system}

The objectives of the IDSS upgraded from the Proactive Ageing platform are: 
- Implementing intelligent decision support services that can help identify the most appropriate therapeutic protocol for a particular patient;

- Designing and deploying analyticalenabled services targeted to the patient;

- Using Big Data Analytics to process medical and environmental data and information;

- Creating an intelligent environment (smart-environment) centered on the patient both for home and for a clinic / institutionalized environment capable of functioning based on integrated support services;

- Ensuring increased protection for vulnerable patients and ensure their safety by providing real-time location capabilities and blocking access to restricted or unsafe areas.

- Implementing a scalable, flexible, affordable, and portable IT solution that is also financially more efficient and provides more personalized, performant and less intrusive medical records;

- Defining a holistic approach to chronic ageing-related disease based on patient-centered data management;

- Identifying dysfunctions and restrictions in patient care to establish new services that correct them to continually improve patient access to health care and treatment efficacy;

- Raising the quality of health care services by diversifying the capabilities of healthcare professionals and facilitating access to other information and clinical research through cloudcomputing services.

The new components should provide improved services aimed at supporting medical activities adapted to the specifics and requirements of a patient such as correct, preventive and real-time diagnosis, or identifying and establishing a personalized therapeutic protocol adapted to possible changes in the course of a chronic ageing-related disease or induced by the occurrence of comorbidities.

The system should allow the patientrelated data obtained with medical devices and intelligent technologies to be centralized, analyzed and processed. Thus, the medical staff, personal caregivers and families are empowered with the ability of tracking the real patient's health status (for example the evolution of some medical parameters) and defining a personalized patient profile and medical services tailored to his needs and peculiarities for both institutionalized and remote care.

The system aims at a complex monitoring of the health status of a patient (such as an elderly person) both at home and in an institutionalized clinic (nursing home or clinic). Intelligent, patient-centered services obtained as a result of intelligencebased monitoring and processing with innovative technologies should provide personalized, dynamic, portable, flexible, and effective support for:

- Patient (supporting and prolonging an independent, active, worthy life, preventing a faster deterioration of health or serious accidents / incidents, recovering in a familiar environment of post-traumatic incident);

- The data are collected from the patient in real-time and, based on these data, the IDSS creates a patient profile through the process of machine learning. An alert is triggered when one or more values within the profile get out of range. (see Table 2). 
Table 2: Examples of alerts triggered by the system

\begin{tabular}{|l|l|l|}
\hline \multicolumn{1}{|c|}{ Reading name } & \multicolumn{1}{|c|}{$\begin{array}{c}\text { Average reading } \\
\text { values }\end{array}$} & \multicolumn{1}{c|}{ Scenarios and IDSS intervention } \\
\hline $\begin{array}{l}\text { Blood Pressure } \\
\text { (BP) }\end{array}$ & $\begin{array}{l}\text { Systolic pressure (SBP) } \\
\text { [mmHg] } \\
\text { Diastolic pressure } \\
\text { (DBP) [mmHg] }\end{array}$ & $\begin{array}{l}\text { If SBP }>=120 \text { and if DBP }>=70 \text { then the } \\
\text { system triggers an alert of a higher than } \\
\text { normal reading. } \\
\text { If SBP }<=90 \text { and DBP }<=45 \text { then the } \\
\text { system triggers an alert of a lower than } \\
\text { normal reading. }\end{array}$ \\
\hline Heart Rate (HR) & BPM [Beats Per Minute] $]$ & $\begin{array}{l}\text { If HR }>=80 \text { then the system triggers an } \\
\text { alert of a higher than normal reading. } \\
\text { If HR }<=50 \text { then the system triggers an } \\
\text { alert of a lower than normal reading. }\end{array}$ \\
\hline
\end{tabular}

- Healthcare staff (constant monitoring of health status, therapeutic protocols, resources consumed for care; a personalized management of the patient's health; creation of a framework for the development of personalized proactive models of a particular disease or co-morbidity; raising the level of professional knowledge);

- Personal caregivers and patient's family;

- Clinical environment (more efficient monitoring of an institutionalized patient - physiological parameters, treatment, consumption of medical resources, patient safety and location, a modern interface with the patient and provision of healthcare services based on new and innovative digital technologies).

- Health system and the social system (avoiding unnecessary institutionalizations, diminishing the consumption of financial, material and human resources by preventing accidents or deterioration of health, facilitating ways to migrate the system to personalized, affordable, patientcentered care).

For collecting data from sensors, the new components will implement the "sensing as a service (SNaaS)" concept, developed by ICI Bucharest. By implementing the described concept, it is provided a unique interface for the beneficiaries of data from sensors - medical units or patients, based on the services integrator business model.

\section{The architecture of proposed real-time IDSS system}

The architecture includes three main layers (see Figure 3):

- Sensing Layer: RFID physical components and IoT monitoring sensors collect information from the targeted patients and capture the data related to health status and movement from them. Thus results in a huge amount of data (Big Data).

- Middleware Layer: translates the raw data from the physical layer to the context (health related conditions, position and movement of the person). Here are included procedures for data cleansing and fusion. The data is stored in data storage volumes.

- Service Layer: the stored information about the tracked individuals is analyzed (using BDA), processed and made accessible to medical staff and caregivers by using queries throughout real time monitoring servers. Notifications or warnings are provided in case of risks to the caregivers through monitoring and assessment consoles. 


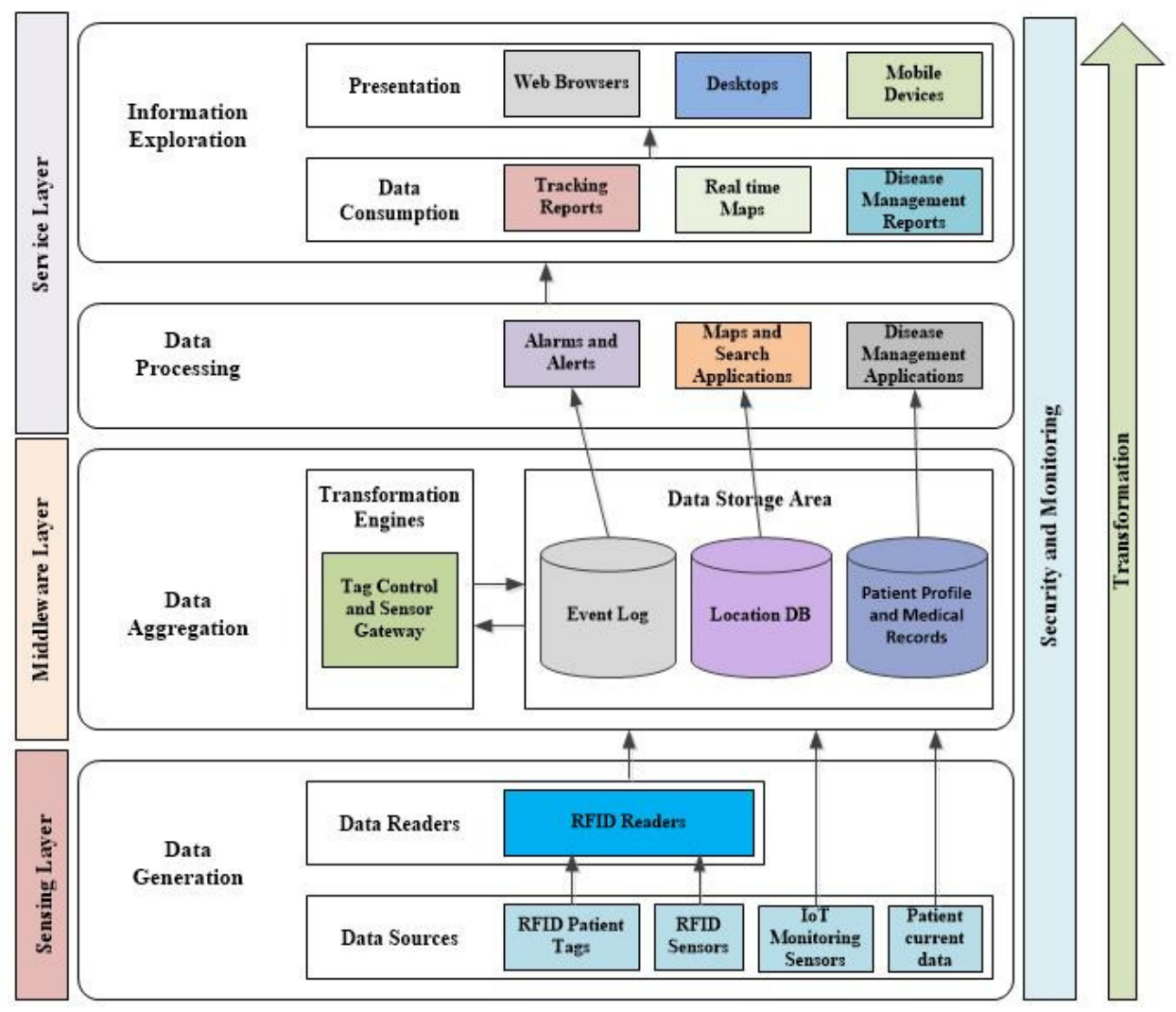

Fig. 3: The IDSS system architecture

\section{The proposed outcomes of the IDSS system}

The proposed outcomes of the IDSS system are referring to:

- Personalized and patient-centered integrated services:

- Physiological parameter monitoring services: should be able to facilitate the measurement of some health parameters, the collection of data and information on the physical and mental state of the patient through the use of IoT technologies. The data can be stored in a patient-centered database for updating personal medical history and the development of predictive models;

- Diagnostics services based on remote monitoring;
- $\quad$ is designed to request for support to a career / family member in case of an unexpected event;

- Daytime assistance services: such as identifying a lost object by activating an online feature, which, by using a wireless sensor, triggers a beep. Another feature of this component is Physical accident or environmental malfunction alerting services: by activating IoT sensors; a warning application will be initiated, both locally and remotely.

- Proactive care services: based on data and information gathered through monitoring, new proactive services can be developed to allow a personalized management of a permanently variable health condition. Thus, they should facilitate an early identification of symptoms that announce the onset 
of a health crunch, followed by the application of countermeasures.

- Support services for informal caregivers and the creation of a personalized environment based on the patient's specificities: provide upto-date and practical information for caregivers to improve the health and well-being of their patients, as well as to adapt housing to the patient needs;

- Patient-embedded monitoring systems customized to existing co-morbidities: e.g. a foot-mounted sensing device that measures motion and travel angle combined with IoT sensors that measure heart rate / voltage, etc.

\section{Conclusions}

Providing a new approach for the Romanian healthcare domain, the proposed IDSS system offers:

- Secure data exchange and interoperability for sensitive data;

- Flexible identity management for creating anonymization while providing customized services;

- Distributed data processing on available network and cloud components;

- Sensors, actuators and hubs that are compatible and interoperable within the defined framework;

- Tools and methods for creating and evaluating services that use these innovations;

- Data cleansing and fusion methods and reliability assessment for the use of heterogeneous data by different actors and entities (e.g., mobile data sources).

- Data analysis on both sensor and service side.

- Cloud connection security.

Turning the ProActive Ageing integrated platform for online services into an Intelligent Decision Support System represents a practical demonstration of how a health informatics system can have a strong social impact on daily life, health and independence of seniors, caregivers education (Alexandru et al., 2007) and last, but not least, on the financial, human and material resources involved by the social and healthcare systems in dealing with the ageing population.

\section{Acknowledgements}

"PROlonging ACTIVE life for an independent and healthy AGEING" (ProActive Ageing) project is funded by the Romanian Partnership Program, Joint Applied Research Projects Subprogram under the National Plan for Research, Development and Innovation (2014-2017).

"Innovative platform for personalized monitoring and support in e-Health" project PN 1819-03-01 is a research project developed inside the Romanian Core Program of the Ministry of Research and Innovation (MCI), 2018.

\section{References}

1. Alexandru, A., Ianculescu, M., Parvan, M. and Jitaru, E. (2007) 'ICT and its Impact upon the globalization and Accessibility of the Education in the Health Domain', $6^{\text {th }}$ WSEAS International Conference on Education and Educational Technology, Italy, 11/21, 287-291.

2. Basu, R., Archer, N. and Mukherjee, B. (2012) 'Intelligent decision support in healthcare', Analytics, 33(38).

3. Foster, D., Mcgregor, C. and El-Masri, S. (2005) 'A survey of agent-based intelligent decision support systems to support clinical management and research', Proceedings of the 2nd International Workshop on Multi-Agent Systems for Medicine, Computational Biology, and Bioinformatics, 16-34.

4. Ianculescu, M. and Alexandru, A (2016) 'Silver Digital Patient, a New Emerging Stakeholder in Current Healthcare. ProActiveAgeing: A Case Study', Studies in Informatics and Control, 25(4), 461-468.

5. Ianculescu, M., Alexandru, A. and Gheorghe-Moisii, M. (2017a) 'Harnessing The Potential of Big Data In Romanian Healthcare', Proceedings of The 5th International Symposium on Electrical and 
Electronics Engineering (ISEEE), Galati, Romania, 20 - 22 Oct.

6. Ianculescu, M., Stanciu, A., Bica, O. and Neagu, G. (2017b) 'Innovative, Adapted Online Services that Can Support the Active, Healthy and Independent Living of Ageing People. A Case Study', International Journal of Economics and Management Systems, 2.

7. IBM (2012) The value of analytics in healthcare, IBM Global Business Services.

8. IERC (2014) European Research Cluster on the Internet of Things Cluster, [Online]http://www.internet-of-thingsresearch.eu/about_iot.htm.

9. Kagermann, H., Wahlster, W. and Helbig, J. (2013) 'Recommendations for implementing the strategic initiative INDUSTRIE' 4.0, Final report of the Industrie 4.0 Working Group.

10.Mantea, TA., Alexandru, TG., Pupăză, C. and Nicolescu, AF. (2016) 'Software Integration for Heat Transfer Simulation of
Electronic Circuits', Studies in Informatics and Control, ISSN 1220-1766, 25(1), 69-76.

11. Manyika, J., Chui, M.,Brown, B., Bughin, J., Dobbs, R,; Roxburgh, C. and Byers, AH. (2011) Big data: The next frontier for innovation, competition, and productivity.

12.Neagu, G., Florian V., Stanciu A. and Preda S. (2016) "Sensing as a service approach in health monitoring.", 15th RoEduNet Conference: Networking in Education and Research, 1-5.

13.Portela, F., Santos, MF., Machado, J., Abelha, A., Silva, Á. and Rua, F. (2014) 'Pervasive and intelligent decision support in intensive medicine-the complete picture', International Conference on Information Technology in Bio-and Medical Informatics, 87-102, Springer, Cham.

14.Viademonte, S. and Burstein, F. (2006) 'From knowledge discovery to computational intelligence: A framework for intelligent decision support systems', Chapter 4 in Intelligent Decision-making Support Sytems, Springer-Verlag London Limited, 57-78. 